\title{
Probable Treatment Options against Infections with Bacteria Carrying bla ${ }_{\mathrm{NDM}-1}$ Gene
}

\author{
Anuj Sud $^{1 *}$, Beena Uppal ${ }^{2}$, Swati Sharma $^{3}$, Prabhav Aggarwal $^{4}$ and Rakesh Kumar \\ ${ }^{1}$ Department of Microbiology, Lok Nayak Hospital and Maulana Azad Medical College, \\ New Delhi-110002, India \\ ${ }^{2}$ Department of Microbiology, Dr Baba Saheb Ambedkar Medical College, Sector-6, \\ Rohini, New Delhi-110085, India \\ ${ }^{3}$ Department of Microbiology, Rajiv Gandhi Superspeciality Hospital, Tahirpur, Delhi, India \\ ${ }^{4}$ Department of Microbiology, ESI Dental College, Sector-15, Rohini, New Delhi-110089, India \\ ${ }^{5}$ Department of Anaesthesiology and Critical Care, Maulana Azad Medical College and \\ Associated Lok Nayak Hospital, New Delhi-110002, India \\ *Corresponding author
}

\section{A B S T R A C T}

\begin{tabular}{|l|}
\hline Ke y w o r d s \\
bla $a_{\mathrm{NDM}-1}$ gene, NDM-1 \\
producing bacteria, \\
NDM-1 treatment, \\
Multidrug resistance, \\
Hospital acquired \\
infection.
\end{tabular}

\section{Keywords}

bla $_{\mathrm{NDM}-1}$ gene, NDM-1 producing bacteria, Multidrug resistance, Hospital acquired

\section{Introduction}

Hospital acquired infections or nosocomial infections are those which develop 48 hours after hospital admission or within 48 hours after being discharged that was not incubating at the time of admission at hospital (Ferrer et al., 2008). Some factors which increase the risk of nosocomial infections are inadequate environmental hygienic conditions and waste disposal, poor infrastructure, insufficient equipments, understaffing, over crowding,
Bacteria have different mechanisms by which they become resistant to antimicrobials like reduced uptake, efflux pumps, drug inactivating enzymes like $\beta$-lactamases etc. New Delhi Metallo- $\beta$-lactamase-1 (NDM-1) is an enzyme which can break down and destroy multiple classes of antibiotics. Infections with multidrug resistant organisms like those carrying bla $a_{\mathrm{NDM}-1}$ gene can lead to adverse outcomes esp. in critically ill patients, patients undergoing organ transplant and other major surgeries. The treatment options are quite limited with the main options available for sometime being limited to colistin and ticecycline which have side effects like renal toxicity. Antimicrobial susceptibility testing VITEK-2 (biomerieux $\left.{ }^{\circledR}\right)$ ofNDM-1 producing bacteria from the ICU admitted showed that nitrofurantoin, gentamicin, tobramycin, levofloxacin and trimethoprim/sulfamethoxazole combination were effective against some strains carrying the gene.

poor knowledge and application of basic infection control measures, lack of knowledge of injection and blood transfusion safety, and absence of local and national guidelines and policies (WHO, 2013). ICU is a suitable place for nosocomial infections due to high antibiotic use, invasive procedures, prolonged hospital stay, critically ill patients, cross transmission among patients and staff, and inadequate infection control procedures (Playford et al., 2013; Weber et al., 2007; Fournier et al., 2007). Studies have shown the 
higher occurrence rates for most antimicrobial resistant organisms in ICU compared to non ICU patients which leads to higher morbidity and mortality in critically ill patients (Mohanasoundaram, 2010).

NDM-1 was first isolated from a Swedish patient who had a history of hospitalisation in India (New Delhi) and thus it came to be known as New Delhi Metallo- $\beta$-lactamase-1 based on the city of origin. NDM-1 is an enzyme belonging to Ambler Class B Metallo- $\beta$-lactamase since it contains zinc at active site. NDM-1 producing Enterobacteriaceae have been found to cause bacteremia, ventilator associated pneumonia, soft tissue and urinary tract infections and limited treatment options have resulted in poor outcomes in patients infected with these multidrug resistant bacteria.

The study was carried out on the Gram negative bacterial strains obtained from culture of blood, urine and tracheal aspirates of patients admitted to the ICU of tertiary care hospital in New Delhi between December 2013 and January 2015. A total of 140 patients were enrolled in the study and follow up was also done. Samples were collected on three occasions- 0-2 days, 3-7 days and >7 days of ICU stay. Culture was done as per standard protocol and bacteria obtained were screened for carbapenem resistance using $10 \mu \mathrm{g}$ disc of meropenem. Production of metallo- $\beta$-lactamase was confirmed by double disc synergy test using Imipenem and Imipenem-EDTA discs. Strains found to be producing Metallo- $\beta$-lactamase were tested for detection of bla $a_{\mathrm{NDM}-1}$ gene by using Real time PCR (SYBR green).

\section{Results and Discussion}

A total of 184 bacteria were isolated during the study and 34 of them were found to be producing NDM-1. Similar isolates from different samples of a single patient were considered as one to avoid duplication. 20 NDM-1 producing bacteria were obtained from tracheal aspirate samples while 8 and 6 strains were obtained from urine and blood samples respectively. The strains comprised of- 9 Klebsiella pneumoniae, 15 Acinetobacter baumannii, 8 Escherichia coli, 1 each of Enterobacter cloacae and Proteus mirabilis. Antimicrobial susceptibility testing was carried out using VITEK-2 system (biomerieux $®$ ).

The antibiotics that were tested includedampicillin, amoxycillin + clavulanic acid, ampicillin + sulbactam, piperacillin + tazobactam, cefazolin, ceftazidime, ceftriaxone, cefepime, ertapenem, imipenem, gentamicin, tobramycin, ciprofloxacin, levofloxacin, nitrofurantoin, trimethoprim + sulfamethoxazole. 20 strains were found to be resistant to all the antibiotics tested by VITEK-2. However, 14 strains did show some susceptibility to a few antibiotics. (Table 1).

Among the isolates from tracheal aspirates, one each of E.coli and Acinetobacter baumannii strain showed intermediate susceptibility to levofloxacin while another $E$. coli strain was found to be susceptible to gentamicin and tobramycin. Two strains of $E$. coli were found to be susceptible to nitrofurantoin while one more strain of $E$. coli and Klebsiella pneumoniae each were found to be intermediate susceptible. The susceptibility to levofloxacin, gentamicin and tobramycin in the isolates from tracheal aspirates is significant as these drugs are useful in treating pulmonary infections while the same cannot be said about Nitrofurantoin since CLSI recommends the use of Nitrofurantoin for treating urinary tract infections mainly.

From the isolates obtained from the urine 
sample, two E. coli isolates were sensitive to nitrofurantoin and among them one was sensitive to gentamicin and tobramycin while another $E$. coli isolate which was intermediate susceptible to nitrofurantoin was susceptible to these drugs. Three strains of Acinetobacter baumannii were found to be intermediate susceptible to levofloxacin while one of them was also sensitive to tobramycin. The Enterobacter cloacae strain was sensitive to trimethoprim-sulfamethoxazole combination. Use of nitrofurantoin to treat urinary tract infections has been documented in the study by Rogers et al., where UTI with NDM-1 positive $E$. coli was successfully treated as the patient showed negative follow up urine samples (Rogers et al., 2017). Other antibiotics like gentamicin, tobramycin and levofloxacin have also been recommended to treat infections caused by these bacteria.

An E. coli strain from blood was found to be sensitive to trimethoprim/sulfamethoxazole combination while one Klebsiella and one Acinetobacter strain were found to be intermediate sensitive to levofloxacin and tobramycin respectively. Whether the antibiotics which have shown efficacy in vitro against NDM-1 producing bacteria are effective in vivo remains to be seen since clinical studies showing efficacy of these antibiotics against the multidrug resistant NDM-1 producers are lacking.

Another problem encountered with these bacteria is the problem of pan drug resistance where it shows resistance to all the antibiotics available and tested. In such cases, combination therapy should be used since successful treatment has been carried out with combination therapies as shown by Wilowski et al., where the patients who received imipenem/cilastatin, colistin and fosfomycin combination did not show any NDM-1 positive bacteria in follow up urine samples (Wilkowski et al., 2016). However, antimicrobial susceptibility testing of all NDM-1 positive strains should be carried out and the antibiotics found effective in vitro should be tried especially in those cases where other higher antibiotics are contraindicated.

\section{References}

Ferrer M, Valencia M, Torres A. Management of ventilator associated pneumonia. In: Vincent JL, ed. 2008 Year Book of Intensive Care and Emergency Medicine. Verlag Berlin Heidelberg: Springer; 2008: e353-364.

Fournier PE, Richet H. The epidemiology and control of Acinetobacter baumannii in health care facilities. Clin Infect Dis. 2006 Mar 1; 42: 692-9.

Mohanasoundaram KM. Retrospective analysis of incidence of nosocomial infections in ICU. J Clin Diagn Res. 2010 Dec;4: 3378-82.

Playford EG,Craig JC, Iredell JR. Carbapenem-resistant Acinetobacter baumannii in intensive care unit patients: risk factors for acquisition, infection and their consequences. $\mathbf{J}$ Hosp Infect. 2007 Mar; 65: 204-11.

Rogers BA, Sidjabat HE, SilveyA, Anderson TL, Perera S, Li J et al., Treatment options for New Delhi metallo betalactamase harboring enterobacteriaceae. Microb Drug Resist. 2013 Apr; 19: 100-3.

Weber DJ, Rutala WA, Sickbert-Bennett EE, Samsa GP, Brown V, Niederman MS. Microbiology of ventilator associated pneumonia compared with that of hospital-acquired pneumonia. Infect Control Hosp Epidemiol. 2007 Jul; 28: 825-31.

Wilkowski P, Ciszek M, Dobrzaniecka K, Sańko-Resmer J, Łabuś A, Grygiel K et al., Successful Treatment of Urinary Tract Infection in Kidney Transplant 
Recipients Caused by Multiresistant Klebsiella pneumoniae Producing New Delhi Metallo-Beta-Lactamase (NDM1) With Strains Genotyping. Transplant Proc. 2016 Jun; 48: 1576-9.
Available from: http://www.who. int/gpsc/country_work/gpsc_ccisc_fact _sheet_en.pdf. Accessed February 10,2013

World Health Organization. Health careassociated infections fact sheet. 2013.

\section{How to cite this article:}

Anuj Sud, Beena Uppal, Swati Sharma, Prabhav Aggarwal and Rakesh Kumar. 2017. Probable Treatment Options against Infections with Bacteria Carrying bla $a_{\mathrm{NDM}-1}$ Gene. Int.J.Curr.Microbiol.App.Sci. 6(12): 2803-2806. doi: https://doi.org/10.20546/ijcmas.2017.612.325 